\title{
PATIENT-PROSTHESIS MISMATCH IS A PREVENTABLE DISEASE BUT HOW TO PREVENT IT IS A STORY NOT YET WRITTEN
}

\author{
Antonio Calafiore ${ }^{1}$, Antonio Totaro ${ }^{1}$, Stefano Guarracini ${ }^{2}$, Sotirios Prapas ${ }^{3}$, Massimo Di \\ Marco $^{4}$, Kostas katsavrias ${ }^{3}$, Mario Gaudino $^{5}$, Roberto Lorusso ${ }^{6}$, and Michele Di Mauro ${ }^{6}$ \\ ${ }^{1}$ Gemelli Molise \\ ${ }^{2}$ Private Hospital Pierangeli Srl \\ ${ }^{3}$ Henry Dunant Hospital Center \\ ${ }^{4}$ Ospedale Civile dello Spirito Santo \\ ${ }^{5}$ NewYork-Presbyterian Hospital/Weill Cornell Medical Center \\ ${ }^{6}$ Maastricht UMC+
}

December 30, 2020

\begin{abstract}
Large studies demonstrated that moderate or severe patient-prosthesis mismatch (PPM) occurs in $44.2 \%$ to $65 \%$ of patients undergoing aortic valve replacement. If there is general agreement that patients with PPM have worse outcome than patients without, it is difficult to understand how to prevent this dangerous complication. The formula used to calculate the effective orifice area (EOA) of an implanted aortic prosthesis has many weak points that produce inconsistent results using the same prosthetic valve (type and size). The observed EOA (3 to 6 months postoperatively) of a \#23 biological prosthesis can range from 0.9 to $3.5 \mathrm{~cm}^{2}$, making PPM prevention impossible using projected EOA, where only the mean value is reported $\left(1.83 \mathrm{~cm}^{2}\right.$ for the same \#23 biological prosthesis). An EACTS-STS-AATS Valve Labelling Task Force has been established to suggest the manufacturers to present essential information on valvular prosthesis characteristics in standardized Valve Charts. For valves used in the aortic position, Valve Charts should include a standardized PPM chart to assess the probability of PPM after implantation. This will not solve completely the conundrum of prevention, but most likely it will be a step ahead.
\end{abstract}

\section{PATIENT-PROSTHESIS MISMATCH IS A PREVENTABLE DISEASE BUT HOW TO PREVENT IT IS A STORY NOT YET WRITTEN}

Antonio M. Calafiore (1), MD, Antonio Totaro (1), MD, Stefano Guarracini (2), MD, PhD, Sotirios Prapas (3), MD, Massimo Di Marco (4), MD, Kostas Katsavrias (3), MD, Mario Gaudino (5), MD, Roberto Lorusso (6), MD, PhD, Michele Di Mauro (2) (6), MD, PhD

1. Department of Cardiovascular Sciences, Gemelli Molise, Campobasso, Italy

2. Department of Cardiology, "Pierangeli" Hospital, Pescara, Italy

3. Department of Cardiac Surgery, Henry Durant Hospital, Athens, Greece

4. Department of Cardiology, "Santo Spirito" Hospital, Pescara, Italy

5. Department of Cardiothoracic Surgery, Weill Cornell Medicine, New York, NY, USA

6. Cardio-Thoracic Surgery Unit, Heart and Vascular Centre, Maastricht University Medical Centre (MUMC), Cardiovascular Research Institute Maastricht (CARIM), Maastricht, The Netherlands

\section{Corresponding author}


Antonio M. Calafiore, MD

Department of Cardiovascular Diseases

Gemelli Molise

Largo A. Gemelli, 1

86100 Campobasso, Italy

email:am.calafiore@gmail.com

\begin{abstract}
Large studies demonstrated that moderate or severe patient-prosthesis mismatch (PPM) occurs in $44.2 \%$ to $65 \%$ of patients undergoing aortic valve replacement. If there is general agreement that patients with PPM have worse outcome than patients without, it is difficult to understand how to prevent this dangerous complication. The formula used to calculate the effective orifice area (EOA) of an implanted aortic prosthesis has many weak points that produce inconsistent results using the same prosthetic valve (type and size). The observed EOA (3 to 6 months postoperatively) of a \#23 biological prosthesis can range from 0.9 to 3.5 $\mathrm{cm}^{2}$, making PPM prevention impossible using projected EOA, where only the mean value is reported (1.83 $\mathrm{cm}^{2}$ for the same \#23 biological prosthesis). An EACTS-STS-AATS Valve Labelling Task Force has been established to suggest the manufacturers to present essential information on valvular prosthesis characteristics in standardized Valve Charts. For valves used in the aortic position, Valve Charts should include a standardized PPM chart to assess the probability of PPM after implantation. This will not solve completely the conundrum of prevention, but most likely it will be a step ahead.

In this issue of the Journal, Luthra et al. ${ }^{1}$, using the effective orifice area (EOA) as provided by the manufacturers, found that long-term survival in patients who underwent aortic valve replacement (AVR) was influenced by the absolute EOA or indexed EOA (iEOA). Patients with moderate or severe prosthetic stenosis (EOA [?]1.5 cm2) had globally a lower survival than patients without (when AVR was performed by a tissue valve, but not if a mechanical prosthesis was used). Patients with moderate or severe patient-prosthesis mismatch (PPM) (iEOA [?]0.85 $\mathrm{cm}^{2} / \mathrm{m} 2$ ) had a lower survival than patients without.
\end{abstract}

There is general agreement that a significant prosthetic stenosis can be a disease that can affect patients' quantity and quality of life. However, in order to prevent PPM, when an aortic valve is replaced by a certain type of prosthesis of a given size, we need to know in the surgical theatre if we are imposing a disease similar to that we are treating. For this purpose, before starting a case, we consult the projected prosthetic iEOA charts to select the appropriate valve size in order to predict and prevent PPM. It is common experience that those charts are often unreliable, as in large studies moderate or severe PPM occurs in $44.2 \%^{2}, 53.7 \%^{3}$ and $65 \%^{4}$ of the patients.

The EOA of a prosthesis is calculated applying the concept of the continuity equation that the stroke volume (SV) ejected through the left ventricular outflow tract (LVOT) all passes through the aortic valve area (AVA) and thus SV is equal at both sites: $\mathrm{SV}(\mathrm{AV})=\mathrm{SV}(\mathrm{LVOT})$. Because volume flow rate through any cross-sectional area (CSA) is equal to the CSA multiplied by the flow velocity time integral (VTI) over the ejection period, this equation can be rewritten as: AVA*VTI $(\mathrm{AV})=\mathrm{CSA}(\mathrm{LVOT}) * \mathrm{VTI}(\mathrm{LVOT})$. Solving for AVA yields the continuity equation AVA $=\mathrm{CSA}(\mathrm{LVOT}) * \mathrm{VTI}(\mathrm{LVOT}) / \mathrm{VTI}(\mathrm{AV})$. Calculation of continuityequation valve area then requires three measurements: AVA jet velocity, LVOT diameter for calculation of a circular CSA, and LVOT jet velocity.

The greatest potential source of error in the continuity equation is the CSA of the LVOT. It is assumed that LVOT is circular. However, it should be remembered that LVOT becomes progressively more elliptical (rather than circular) in many patients, which may result in underestimation of its CSA (squared in the equation) and in subsequent underestimation of SV and eventually AVA. The location of LVOT measurement is as well important. LVOT diameter has to be measured as close as possible to the aortic annulus, whereas 
measurements done 5 to $10 \mathrm{~mm}$ below yield significant underestimation of the stroke volume and of the $\mathrm{AVA}^{5}$.

The phenomenon of pressure recovery is another problem that affects flow velocity through the aortic prosthesis (in the continuity equation this value is at the denominator: the higher the lower the EOA). Convergence of flow through the stenotic aortic valve to the vena contracta converts potential energy to kinetic energy with a resulting reduction in pressure at the vena contracta ${ }^{6}$. As streamlines then diverge and slow again distal to the vena contracta, there is reconversion of some kinetic energy to potential energy with recovery of a proportion of the pressure lost from the LVOT to the vena contracta ${ }^{7}$. Because Doppler-based methods detect peak flow velocity that occurs at the vena contracta, the transvalvular pressure drop estimated by Doppler will be greater than that calculated from simultaneous invasive pressure measurements in the LVOT and in the aortic root distal to the vena contracta. Pressure recovery, at the same EOA, is inversely proportional to the diameter of the ascending aorta, as it is less pronounced when the proximal aorta is dilated because energy is lost from nonlaminar flow and turbulence ${ }^{8,9}$. Measurement of AVA using echocardiography does not account for aortic size and the degree of pressure recovery and, therefore, may overestimate the severity of prosthetic stenosis ${ }^{10}$.

Another method, intuitively more accurate and reproducible, is the predicted iEOA, using the size and the model of the prosthesis divided by body surface area (BSA). BSA depends on a constant (height) and a variable (weight). It is very likely the BSA calculated with the lean mass should be used, being the metabolic needs of the fat tissue very small. This concept is present in the literature, as PPM in patients with a body mass index $>30 \mathrm{~kg} / \mathrm{m}^{2}$ seems not to be a risk factor for survival ${ }^{11}$. Recent guidelines suggest lower threshold for PPM in obese patients ${ }^{12,13}$.

All the problems in the correct evaluation of EOA and iEOA are evident in several studies where patients with the same prosthesis (Carpentier-Edwards Perimount \#23, Irvine, CA, USA) showed a mean EOA of $1.83+-0.413 \mathrm{~cm} 2$, the EOA ranging from 0.9 to $3.5 \mathrm{~cm} 2^{14}$. In the reference table, however, only the mean value of $1.83 \mathrm{~cm} 2$ is incorporated. In the PERIGON study, the EOA measured at 3-6 months after surgery showed that there was a wide range of EOAs for the same prosthesis (Avalus \#23, Medtronic, Minneapolis, MN, USA). The EOAs ranged from 0.92 to $2.40 \mathrm{~cm} 2$ with a mean value of $1.51+-0.3 \mathrm{~cm} 2$. Comparing the results of the measured iEOAs with the projected iEOAs, the Authors found that the use of an iEOA chart led to the incorrect prediction of PPM in $30 \%$ of the patients and severe PPM in $22 \%$ of the patients ${ }^{15}$.

The fact that so many patients are misclassified as having PPM, based on projected EOA values, is also relevant for the interpretation of studies that examined the effect of PPM on survival. The majority of studies use reference EOAs derived from the literature to calculate projected iEOA values and determine the presence of $\mathrm{PPM}^{2-4}$. The impact on long-term outcomes of PPM based on projected EOA, or the lack of an impact, may be confounded by misclassification bias.

The solution to this conundrum is not easy. The jet velocity, measured in the operating theatre, is not reliable, as it can be higher or lower according to the patient's anemic status, heart rate and ejection fraction. All these variables will prevent any surgeon to explant a prosthesis with high gradient trying to reimplant a larger one. The fact that the same type and size of prosthetic valve can provide different EOA, with/out PPM, makes PPM prevention difficult, as many components come from the patient and are out of our control. Indexed EOA charts provided by valve manufacturers have been severely criticized for their inaccuracy ${ }^{16}$, being regarded by many as marketing tools rather than useful clinical assets ${ }^{17}$. An EACTSSTS-AATS Valve Labelling Task Force ${ }^{18}$ has been established to suggest the manufacturers to present essential information on valvular prosthesis characteristics in standardized Valve Charts. For valves used in the aortic position, Valve Charts should include a standardized PPM chart to assess the probability of PPM after implantation. A Valve Chart like this would be surely a great improvement, but PPM probability is still provided after dividing patients into the classic categories. A recent study found a near-linear relation between iEOA and mean aortic pressure gradient, a relation that did not differ between patients with and without obesity, suggesting that the use of lower cut-off values of iEOA to classify patients with obesity should be reconsidered ${ }^{19}$. 
The history of how to prevent PPM is not yet written and, perhaps, will never be.

\section{REFERENCES}

1. Luthra S, Malvindi P, Olevano C, Zingale A, Salem H, Ohri S. Impact of valve size, predicted effective and indexed effective orifice area after aortic valve replacement. J Card Surg, 2021, in press.

2. Head SJ, Mokhles MM, Osnabrugge RL, et al. The impact of prosthesis-patient mismatch on long-term survival after aortic valve replacement: a systematic review and meta-analysis of 34 observational studies comprising 27186 patients with 133141 patient-years. Eur Heart J 2012;33:1518-29.

3. Sa M, de Carvalho MMB, Sobral Filho DC, et al. Surgical aortic valve replacement and patient-prosthesis mismatch: a meta-analysis of 108182 patients. Eur J Cardiothorac Surg 2019;56:44-54.

4. Fallon JM, DeSimone JP, Brennan JM, et al. The Incidence and Consequence of Prosthesis-Patient Mismatch After Surgical Aortic Valve Replacement. Ann Thorac Surg 2018;106:14-22.

5. Guzzetti E, Capoulade R, Tastet L, et al. Estimation of Stroke Volume and Aortic Valve Area in Patients with Aortic Stenosis: A Comparison of Echocardiography versus Cardiovascular Magnetic Resonance. J Am Soc Echocard 2020;33:953-63 e5.

6. Clark C. Relation between pressure difference across the aortic valve and left ventricular outflow. Cardiovasc Res 1978;12:276-87.

7. Levine RA, Jimoh A, Cape EG, McMillan S, Yoganathan AP, Weyman AE. Pressure recovery distal to a stenosis: potential cause of gradient "overestimation" by Doppler echocardiography. J Am Coll Cardiol $1989 ; 13: 706-15$.

8. Garcia D, Pibarot P, Dumesnil JG, Sakr F, Durand LG. Assessment of aortic valve stenosis severity: A new index based on the energy loss concept. Circulation 2000;101:765-71.

9. Garcia D, Dumesnil JG, Durand LG, Kadem L, Pibarot P. Discrepancies between catheter and Doppler estimates of valve effective orifice area can be predicted from the pressure recovery phenomenon: practical implications with regard to quantification of aortic stenosis severity. J Am Coll Cardiol 2003;41:435-42.

10. Bach DS, Schmitz C, Dohmen G, Aaronson KD, Steinseifer U, Kleine P. In vitro assessment of prosthesis type and pressure recovery characteristics: Doppler echocardiography overestimation of bileaflet mechanical and bioprosthetic aortic valve gradients. J Thorac Cardiovasc Surg 2012;144:453-8.

11. Mohty D, Dumesnil JG, Echahidi N, et al. Impact of prosthesis-patient mismatch on long-term survival after aortic valve replacement: influence of age, obesity, and left ventricular dysfunction. J Am Coll Cardiol 2009;53:39-47.

12. Kappetein AP, Head SJ, Genereux P, et al. Updated standardized endpoint definitions for transcatheter aortic valve implantation: the Valve Academic Research Consortium-2 consensus document (VARC-2). Eur J Cardiothorac Surg 2012;42:S45-60.

13. Lancellotti P, Pibarot P, Chambers J, et al. Recommendations for the imaging assessment of prosthetic heart valves: a report from the European Association of Cardiovascular Imaging endorsed by the Chinese Society of Echocardiography, the Inter-American Society of Echocardiography, and the Brazilian Department of Cardiovascular Imaging. Eur Heart J Cardiovasc Imag 2016;17:589-90.

14. Bleiziffer S, Ali A, Hettich IM, et al. Impact of the indexed effective orifice area on mid-term cardiacrelated mortality after aortic valve replacement. Heart 2010;96:865-71.

15. Vriesendorp MD, Van Wijngaarden R, Head SJ, et al. The fallacy of indexed effective orifice area charts to predict prosthesis-patient mismatch after prosthesis implantation. Eur Heart J Cardiovasc Imag 2020;21:1116-22. 
16. Bleiziffer S, Eichinger WB, Hettich I, et al. Prediction of valve prosthesis-patient mismatch prior to aortic valve replacement: which is the best method? Heart 2007;93:615-20.

17. Cohen RG, Bourne ET. Industry-generated charts for the selection of stented aortic valve prostheses: clinical tool or marketing ploy? Ann Thorac Surg 2011;91:1001-2.

18. Durko AP, Pibarot P, Atluri P, et al. Essential information on surgical heart valve characteristics for optimal valve prosthesis selection: Expert consensus document from the European Association for CardioThoracic Surgery (EACTS)-The Society of Thoracic Surgeons (STS)-American Association for Thoracic Surgery (AATS) Valve Labelling Task Force. J Thorac Cardiovasc Surg 2020, 10.1016/j.jtcvs.2020.10.001.

19. Vriesendorp MD, Deeb GM, Reardon MJ, et al. Why the categorization of indexed effective orifice area is not justified for the classification of prosthesis-patient mismatch. J Thorac Cardiovasc Surg 2020, 10.1016/j.jtcvs.2020.10.123. 